\title{
$V$ Televisión, nacimiento de un canal autonómico privado en Galicia. Análisis de sus formatos informativos
}

\author{
Alba LóPEz BoLÁs \\ Universidad de Vigo \\ albalopezb29@gmail.com \\ Mercedes Román PoRTAS \\ Universidad de Vigo \\ mroman@uvigo.es
}

\section{Resumen:}

El canal autonómico privado de Galicia, V Televisión, comienza su emisión en mayo del 2010 de la mano del Grupo Voz. En este trabajo se analizan sus formatos informativos con el fin de conocer su capacidad de adaptación al nuevo panorama audiovisual. El objetivo es comprobar por qué fracasó la idea inicial del canal de surgir como un ente principalmente informativo, y en qué medida han conseguido captar a un público específico centralizando la mayor parte de sus recursos en un programa de análisis y debate (Vía V) y con una estrategia efectiva en internet y en las redes sociales.

Palabras clave: V Televisión; formatos informativos; Galicia.

$V$ Televisión, birth of a private regional channel in Galicia. Research of the information formats

\begin{abstract}
:
The private regional channel of Galicia, V Televisión, start broadcasting in May 2010 from the hand of Grupo Voz. In this paper we analyze their information formats in order to determine their capacity to adapt to new audiovisual landscape. The goal is to see why failed the initial channel idea arise mainly as a being informative, and how they have managed to capture a specific audience centralizing most of its resources on a program of analysis and discussion (Vía V) and with an effective strategy on Internet and social networks.
\end{abstract}

Key Words: V Televisión; news formats; Galicia.

\section{Referencia normalizada:}

López Bolás, A. y Román Portas, M. (2014): V Televisión, nacimiento de un canal autonómico privado en Galicia. Análisis de sus formatos informativos. Historia y Comunicación Social. Vol. 19. Núm. Especial Enero. Págs. 367-377.

Sumario: 1. Introducción. 2. Metodología. 3. Análisis del programa de tertulia Vía V. 4. Análisis de los Informativos V Televisión. 5. Estrategia de V Televisión en internet. 5.1. Vía $\mathrm{V}$ en Facebook y Twitter. 6. Conclusiones. 7. Referencias bibliográficas. 8. Anexo: Breve C.V. de las autoras. 


\section{Introducción}

Con el apagón analógico producido en el 2010 en España comienza una nueva era audiovisual. Aumenta la fragmentación televisiva, las alternativas para el espectador y las cadenas creadas para la TDT se multiplican. Se trata de una época complicada, sobre todo, por la crisis económica que se inició en el año 2008 (Román Portas, 2012).

Los cambios en el sector audiovisual gallego comenzaron a fraguarse en el 2005. En julio de este año el Gobierno de la Xunta en funciones adjudicó dos cadenas de televisión digital terrestre de cobertura autonómica (de acuerdo con el Decreto $81 / 2005$ del 14 de abril por el que se regula el régimen jurídico de gestión del servicio público de la televisión digital en la Comunidad Autónoma de Galicia). Una de ellas para La Voz de Galicia S.A. y la otra, a la Radio Popular, S.A., ambas obtuvieron las licencias de TDT por un periodo de diez años. Esta concesión se sumaría a las otras dos licencias de las que ya disponía CRTVG, por lo que, una vez iniciado el apagón analógico en España en el 2010, Galicia tendría cuatro canales autonómicos.

El nuevo canal privado $V$ Televisión del Grupo Voz comienza a emitir el 30 de mayo del 2010. Su adaptación a un sector audiovisual convulso continúa casi tres años después de su puesta en marcha. Hasta principios del 2013 la financiación del canal corre a cargo de la inversión privada de sus socios así como de la publicidad.

La televisión pública, tanto nacional como autonómica, absorbe la mayor parte de la audiencia gallega. En concreto, en 2012 y según datos del EGM, un 54,85\% de la audiencia ha preferido alguno de los dos principales canales públicos antes que los privados generalistas.

Si hay algo que diferencia a la audiencia en Galicia es su apuesta por la información de su comunidad. De hecho, la Televisión de Galicia (TVG) sitúa sus informativos como los más vistos de todas las televisiones autonómicas públicas en España, con una cuota de pantalla que llega a alcanzar el 32,3\% (Miguel de Bustos y Casado del Ríos, 2012). Con todo, la situación se presenta compleja a la hora de poner en marcha un nuevo canal autonómico privado en Galicia.

\section{Metodología}

$V$ Televisión nace con dos señas de identidad claras: la información actualizada cada sesenta minutos a través de boletines de no más de cinco minutos y su orientación hacia un público joven. Pero, a pesar de surgir como una cadena eminentemente informativa, en los dos primeros años de emisión los boletines desaparecen y el espacio dedicado a los Informativos se reduce de forma considerable. Finalmente el canal se queda con un solo informativo diario en prime-time de 30 minutos. 
El objetivo principal del canal no funcionó, por lo que el espacio dedicado a la actualidad se apoya principalmente en el que pronto se convirtió en su programa de referencia, el Vía V, un formato de debate y entrevistas que consigue los mejores datos de audiencia de $V$ Televisión. A lo largo de su corta existencia, este programa de debate y entrevistas ha ido ampliando su duración hasta llegar, en el 2012, a las dos horas y media de programa en directo con una media de share de 0,78 y unos casi 9.000 espectadores cada noche.

Este trabajo pretende comprobar por qué fracasó el modelo informativo inicial del canal centrado en emitir boletines de cinco minutos cada hora y, en cambio, por qué funcionó (desde el punto de vista de la audiencia) el programa de análisis Vía V.

Para cumplir el objetivo principal se han comprobado los datos de audiencia de los programas Vía V desde el inicio de V Televisión (2010) hasta el 2012. Se han seleccionado dos programas mensuales, los de mayor y menor audiencia, para posteriormente, a través de un análisis de contenido, estudiar las razones por las que un programa tiene un determinado seguimiento por los espectadores de la comunidad (Casero Ripollés, 2011). La muestra pertenece al periodo que va desde el 1 de octubre del 2010 hasta el 31 de diciembre del 2012.

En cuanto a la metodología utilizada para el estudio de su presencia en internet se ha clasificado y analizado el tráfico generado en las redes sociales por el programa Vía V y por sus usuarios, y se ha comprobado si repercute de forma directa en el share registrado en cada muestra.

\section{Análisis del programa de tertulia, Vía $\mathrm{V}$}

$V$ Televisión alcanza su mejor cuota de pantalla desde su inicio en mayo de 2011, con un 0,6 de share. Pero a partir de ese momento no consigue una evolución ascendente, sino que oscila entre el 0,8 y el 0,4. Sin embargo, con el programa Vía V no ocurre lo mismo, ya que a partir de 2012 logra picos de audiencia muy significativos que duplican a la media del canal, llegando a alcanzar el 1,5 de share.

Como puntos clave en el programa, además de la entrevista y el debate, se incluye una sección dedicada exclusivamente a internet y a las redes sociales y una sección de noticias que resume y actualiza los titulares de la jornada.

Son importantes los cambios que se empiezan a producir a partir del 2012, cuando el tiempo dedicado a las noticias (resumen de los titulares del día y actualización de los mismos) crece considerablemente, llegando a alcanzar un 15\% del tiempo total y quitándole espacio a la entrevista y al debate, este último promedia entre las tres temporadas un $62 \%$ de tiempo dedicado, sin embargo, mientras que en el año 2010 y 2011 no solía bajar del 80\%, a partir del 2012 oscila entre el $40 \%$ y 60\%. Esto se debe a que también es a partir de este año cuando asciende la sección sobre internet y 
redes sociales, algo que aporta un valor añadido al programa que repercutirá de forma directa en la audiencia.

Aunque pueda parecer que un programa con entrevista también repercutirá positivamente en la audiencia, los datos no lo confirman. De hecho, de los cuatro programas con mayor share de todas las temporadas analizadas, la mitad no introducen una entrevista sino que son en su totalidad un debate entre invitados de diferente índole.

Dos de los programas con mayor audiencia son los que incluyen una entrevista a dos de los personajes políticos más relevantes de la comunidad gallega (Xosé Manuel Beiras y Pachi Vázquez) y se hacen en época de campaña electoral. Son también el caso, aunque con datos inferiores, de la entrevista realizada a Alberto Núñez Feijóo y a Mariano Rajoy. Sin embargo, estos son casos específicos, las cifras demuestran que la realización de una entrevista a un personaje destacado en el inicio del programa no es siempre sinónimo de buena audiencia. De hecho, en el único programa (11 de julio de 2012) de todas las muestras analizadas que supera el 3\% de share, más de un $91 \%$ de su totalidad está dedicado simplemente al debate y a la rueda de prensa del presidente Mariano Rajoy que anuncia las medidas de austeridad más duras que se conocen hasta el momento. En éste y en otros diez casos no hizo falta una entrevista en el programa pero sí unos invitados al debate que fueran capaces, a través de sus argumentos, de mantener audiencias que oscilan entre los 10.000 y los 26.000 espectadores. Se trata de 11 muestras, sin entrevista, en las que existe una repetición en la presencia de varias personas clave en la mayoría de los debates. La aparición reiterada de estos personajes en los programas de mayor éxito de la cadena funciona como un elemento de fidelización importante al comprobar su funcionalidad en cada uno de ellos. Destacan dos personas en particular, ambos colaboradores también de La Voz de Galicia: el catedrático Roberto Blanco Valdés aparece en un 12,7\% de la totalidad de los programas de mayor audiencia, reduciéndose a solo un 4\% su presencia en los de menor audiencia; algo parecido sucede con el profesor Xosé Luis Barreiro con un $8 \%$ frente al $2 \%$ de los programas con menos espectadores.

El ámbito de la política pasa a ser el más relevante cuando se trata de citar al tipo de entrevistados que acuden a los programas Vía V. Sin embargo, aquí sí que encontramos una diferencia: mientras que en los programas de mayor cuota de pantalla un $62 \%$ de los entrevistados son políticos, en los de menor share solo un $46 \%$ son de este tipo, por lo que más de la mitad de los que acuden a las entrevistas son de otro ámbito que alcanzan cuotas muy bajas. Son el caso de los docentes, periodistas o sindicatos.

También es necesario destacar el cargo político de cada uno de los entrevistados en este ámbito. Los programas que alcanzan un share muy alto son aquellos que incluyen una entrevista de personajes conocidos a nivel nacional o con mucho peso autonómico. Por el contrario, los políticos entrevistados en los de menor audiencia son personajes de ámbito local o de menor rango.

Esta tendencia a seleccionar cuestiones nacionales podría extenderse al apartado del debate posterior, sin embargo el estudio muestra una clara apuesta no solo por la actualidad autonómica sino por la variedad en el ámbito de los temas que discuten 
los tertulianos y la presentadora. En los programas con mayor cuota de pantalla de las tres temporadas del Vía V, el tiempo dedicado a la actualidad autonómica ronda el $40 \%$ y hasta en 8 programas supera el 50\% del tiempo total. Además hay tres programas dedicados exclusivamente a la actualidad autonómica. En los programas menos exitosos de las dos temporadas la distribución cambia. La actualidad autonómica pasa a tener un menor peso frente a la nacional y sobre todo la internacional, que supera hasta en seis programas a las otras dos. Esto ocurre sobre todo en la primera temporada de la cadena, por lo que evidencia aún más el mal funcionamiento de temas internacionales en este formato, ya que cuanto menos tiempo se le va dedicando a la actualidad de este tipo mayor es el número de espectadores que alcanza el programa.

Por otra parte, la economía y la política son los bloques más importantes en el programa de análisis de la cadena $V$ Televisión, ya que ocupan un promedio del $87 \%$ del total del contenido del debate. En los programas más vistos, sin embargo, la política con un $53 \%$ de media supera a la etiqueta de economía con un $31 \%$. Por lo que una vez más los temas políticos suponen una clave para captar espectadores. Los temas económicos, aunque también logran picos de audiencia positivos, destacan más por sus datos negativos de share. Esto puede ser debido a lo repetitivo de sus argumentos, en la mayoría de estos casos el tema principal del programa es la deuda de las comunidades autónomas, el paro, la mala situación de Novacaixagalicia o la crisis económica en general. Son conceptos que abren continuamente los informativos y sobre los que ya se empieza a notar un especial hartazgo posiblemente por su connotación negativa.

La evolución del tiempo dedicado a la sociedad es ascendente a lo largo de las temporadas, integrándose como parte pequeña pero importante en el programa a la hora de aportar un aire diferente y temas de alguna forma no tan negativos como pueden ser los políticos o económicos. De hecho, la única emisión (12 de julio de 2011) de todas las analizadas del Vía V que se dedica al $100 \%$ a un tema social trata sobre la posible llegada del AVE a Galicia en el 2015, y se aprovecha para hacer un repaso histórico al ferrocarril en la comunidad gallega. Otros de los temas recurridos en este ámbito son la educación, el Papa o la Casa Real. Asimismo sorprende la ausencia casi por completo de temas de sucesos, algo que también veremos en el análisis de los Informativos y que confirma que la intención de los editores de contenidos no es aproximar la cadena hacia el modelo de programas informativos que se extiende en España, convertidos en términos generales en auténticos magazines (Bustamante, 2011).

En términos generales, se puede decir que el programa Vía $\mathrm{V}$ fue uno de los pilares de la parrilla desde el inicio y funcionó. Debido al contexto económico, $V$ Televisión ha centrado la mayor parte de sus recursos en este programa que ha conseguido ser un referente a la hora de hablar de la actualidad en Galicia. Algo que no se ha conseguido con otros formatos de la cadena. 


\section{Análisis de los informativos V Televisión}

Los Informativos han evolucionado de forma muy diferente a como lo ha hecho el formato de tertulia en $V$ Televisión, y no han conseguido alcanzar datos de audiencia significativos.

En cuanto al tipo de actualidad emitida en los Informativos es muy similar a la del programa Vía $\mathrm{V}$, aunque con la gran diferencia de que la cantidad de temas de actualidad autonómica está en casi la totalidad de las muestras por encima de la nacional e internacional. Por lo demás, también en los telediarios se muestra una clara tendencia a disminuir los contenidos internacionales y a igualar los nacionales con los autonómicos.

Los datos reflejan claramente el amplio espacio que $V$ Televisión le dedica a un acontecimiento como las elecciones autonómicas del 2012. De hecho, en octubre de ese año alcanza su máximo de contenidos de ámbito autonómico con más de un $90 \%$ del total de su informativo. Lo mismo ocurre en mayo de 2011 con las elecciones municipales, momento del que también se aprovecha la cadena para alcanzar altos datos de audiencia gracias a su amplia cobertura por diferentes puntos de Galicia de la campaña electoral y del propio día de la votación.

Si sumamos la información nacional y la internacional oscila en un promedio de entre el $40 \%$ y el $50 \%$ del total del informativo. Este es un resultado que no favorece a la cadena, que nace con el objetivo de contar todo aquello que afecte a la sociedad gallega. Es cierto que también deben cubrir los acontecimientos más importantes que ocurren en el resto del mundo, pero esto puede volverse en su contra al tratarse de una televisión pequeña con muy pocos recursos para todo lo que se intenta cubrir. El espectador no sintoniza $V$ Televisión para ver contenidos de ámbito nacional e internacional, ya que existen otras cadenas que disponen de más medios para ofrecer una mayor cobertura de esta información. Por lo tanto, la posibilidad de que la audiencia cambie de canal cuando está viendo información de su comunidad es mucho menor que con otro tipo de noticias.

A la hora de analizar por secciones la evolución que sufren los Informativos, vuelve a ser mucho más irregular que en el caso del programa de debate de $V$ Televisión. Esto es debido a los numerosos cambios que sufre el formato a lo largo de sus tres años de existencia: cambios de horario, descenso continuado de la duración, creación de secciones que no se mantienen durante toda la temporada, etc.

La tendencia hasta la tercera temporada (septiembre del 2012), cuando el espacio dedicado a los Informativos sufre el mayor recorte en la cadena, es la de aumentar los minutos dedicados a noticias económicas, la gran mayoría marcadas por el peor momento que pasa la crisis económica. Además la política deja cada vez más espacio al ámbito social, que crece desde el 2011 llegando a alcanzar máximos del 40\% del tiempo total del telediario. Los temas políticos, en cambio, van en decadencia desde la segunda temporada hasta el 2012, con excepción del día de las elecciones municipales y las autonómicas cuando alcanzan su máximo de tiempo dedicado con un $48 \%$ 
y un $62 \%$ respectivamente. En cuanto a la cultura y a la autopromoción, se mantienen sobre el 3\% del tiempo total del programa Informativos y se sitúan siempre al final del mismo.

Pero esta estructura sufre un cambio importante, como ya se ha dicho, a partir de la tercera temporada. El espacio dedicado exclusivamente a la economía y al deporte se eliminan y el único informativo que se mantiene es el de prime-time durante solo media hora. Esto repercute de forma negativa en la variedad de contenidos y pasa a ser casi un informativo de economía, política y sociedad, ya que entre los tres superan en la mayoría de los casos el $90 \%$ del tiempo total del telediario.

En cuanto a los sucesos también sufren una caída importante a partir de la temporada 2011-2012 y no vuelven a ganar el peso que tenían en los primeros meses de la cadena cuando llegaban a ocupar el $20 \%$ del total del informativo. Hay incluso muchas muestras en las que el tiempo dedicado a los sucesos es del $0 \%$, algo impensable en muchas cadenas privadas nacionales donde es considerado elemento prioritario dentro de la escaleta.

Más allá del tiempo que se le dedica a los temas, lo importante en un telediario es la jerarquía que se haga de los mismos. En los dos primeros años del canal los temas seleccionados como importantes para los Informativos y el Vía V no coinciden casi nunca, es a partir del 2012 cuando empieza a verse esa coordinación jerárquica entre los dos formatos. A partir de ahí se observa hasta en 16 ocasiones de todas las analizadas que los temas principales que abren el informativo coinciden con los más importantes también en el debate del Vía V. Los más repetidos son de política nacional, corrupción, elecciones y aspectos relacionados con NCG Banco. Excepto los relacionados con la política, todos los demás son temas con mucho peso en una parrilla informativa autonómica, y suelen conseguir cuotas de pantalla muy altas por su alto interés en Galicia.

\section{Estrategia de V Televisión en internet}

Se establecen hasta tres beneficios en el uso de las redes sociales en la difusión de contenidos televisivos (Ortells Badenes, 2012). Atraer nuevos segmentos de la audiencia, fomentar la participación del usuario (el propio ciudadano aporta propuestas temáticas y material audiovisual) y como estrategia para centralizar todas las visitas en el portal multimedia del ente y aumentar los índices de audiencia.

$V$ Televisión interactúa con su público a través de dos redes fundamentales: Facebook y Twitter. Gracias a ellas se forman grupos y comunidades, en las que se difunden e intercambian contenidos escritos y audiovisuales, además de conseguir algo cada vez más difícil en el amplio mundo televisivo: la fidelización de los usuarios. Estos nuevos medios también se convierten en una alternativa para la publicidad, con nuevas fórmulas para el anunciante (González Oñate, 2008). 
El canal $V$ Televisión tiene 12.978 seguidores en Facebook y 10.956 seguidores en Twitter (datos recogidos el 13/10/2013) algo que se podría considerar un éxito dado que la media de audiencia diaria de la cadena no supera los 7.000 espectadores. Sin embargo, si se va más allá de las cifras, se observa que la participación es aún escasa, mientras que la actividad en Twitter se reduce a meros retweets sin tener la capacidad de generar conversación con sus usuarios, en Facebook el comportamiento tampoco es lo suficientemente participativo como debería dado el número de personas que siguen sus publicaciones.

Los resultados son mucho más positivos si los analizamos desde el punto de vista del programa en prime-time de $V$ Televisión. El Vía $\mathrm{V}$ aprovecha todas las posibilidades de las redes sociales y consigue seguidores diferenciados que pueden no identificarse con el canal pero sí con el propio programa.

\subsection{Vía en facebook y twitter}

El programa Vía V ha implementado en la red social Facebook un modelo informativo que repercute directamente en el proceso de captación de espectadores y por lo tanto en sus datos de audiencia. Para el análisis, se han medido varias variables como la participación, la conexión, la dinamización y el tipo de contenidos (Noguera Vivo, 2010).

Resulta interesante el resultado en cuanto a que las muestras que se analizan son aquellas que han conseguido los mejores y los peores resultados de cuota de pantalla de cada mes desde la puesta en marcha del canal en 2010 hasta diciembre del 2012.

La mayor actividad de todas las muestras analizadas se sitúa en el mes de septiembre del 2012, en concreto el día 18 , cuando el 70,5\% del programa fue dedicado a una entrevista al presidente de la Xunta Alberto Núñez Feijoo. En este caso la persona encargada en el programa de la sección de internet consigue con solo la publicación de cuatro entradas generar 68 comentarios y 17 "Me gusta", en total 89 acciones provocadas por un buen uso de la red social. En las cuatro publicaciones no solo se informa al público de lo que ofrecerá el programa sino que se formulan diferentes preguntas para que la audiencia se anime a comentar qué le está pareciendo la entrevista. Además lo que genera más tráfico en Facebook es la siguiente pregunta que se hace a los usuarios: ¿Qué os gustaría preguntar al presidente de la Xunta? Entre todas las cuestiones que formula el público se seleccionan varias a lo largo del programa y una de las redactoras las expone al propio entrevistado en directo. Es una forma clara de introducir a los espectadores en el propio plató a través de su actividad en Facebook, con esto se consigue no solo generar más tráfico en la página del Vía V sino también fidelizar a una audiencia que deja de ser solo espectadora para convertirse por un momento en protagonista.

El caso de la red Twitter es distinto en cuanto al análisis, no tanto en cuanto a resultados. El programa de tertulia se unió a esta red social un año después de su nacimiento, es decir, en junio de 2011. A partir de ahí su actividad también ha ido en 
aumento como en el caso del Facebook del programa pero su evolución no ha sido tan constante, ya que no se consigue mantener una actividad diaria similar.

Para el análisis se comienza por emplear una métrica que cuantifica la relación que existe entre los seguidores y los seguidos de acuerdo con el número de tweets. Se trata del Índice Twitter Following-Followers Ratio (TFF Ratio desarrollado por Dave Donaldson).

El perfil, que recibe el nombre de @ProgramaViaV, acumula 2.108 seguidores (dato recogido el 13/10/2013), un número bastante bajo en relación con otras televisiones de características similares lo que supone a priori poca influencia. Sin embargo, a través del índice TFF la visión es distinta, un resultado del índice del 8,10 indica que el perfil del Vía $V$ en Twitter es líder de su comunidad y que la gente quiere escuchar lo que dice. Por lo tanto, no siempre disponer de muchos seguidores es sinónimo de mayor éxito en la red social, ya que puede romper la cercanía e incluso la credibilidad de una cuenta que debería buscar la comunicación y conversación con su público.

Esto es lo que ocurre con el perfil del canalVTelevisión, a través de su cuenta @ vtelevision que acumula 10.956 seguidores (dato recogido el 13/10/2013), y sigue a 730 usuarios, lo que denota una gran influencia. Pero el TFF ratio es el siguiente: 12,13 , lo que indica, en cambio, poco interés por escuchar a su público.

Por otra parte, desde la creación de su cuenta en esta red social también se han analizado la relación del programa con sus seguidores y el tipo de contenidos que se publican. Para ello se ha seguido un modelo parecido al utilizado para el análisis de Facebook, dividido en conexión, participación, dinamización y actividad total.

Una vez más se confirma que la mayor parte de los programas que alcanzan cuotas elevadas de audiencia también consiguen una buena respuesta en la red. Vía $\mathrm{V}$ es capaz de aprovechar los temas más llamativos para su público y, a través no solo de su promoción sino también de preguntas que introduzcan al espectador en el debate, aumentar el tráfico de contenidos en Twitter.

El día que mejores datos de actividad alcanza es el 19 de marzo de 2012. Ese día se publican 8 tweets, 17 retweets, 2 comentarios de la audiencia y 1 respuesta por parte del programa. La discusión dominante es simplemente la propia opinión de los temas y la causa de tanto tráfico vuelve a ser la posibilidad de hacer preguntas y de comentar sobre la entrevista a un político de relevancia en Galicia. Además se publican a lo largo del programa varios titulares de la entrevista según lo que afirma el entrevistado, por lo que la posibilidad de comentarla a través de la red se hace mucho más fácil para los usuarios. También es una forma de captar a esos espectadores para que sintonicen el programa en directo. 


\section{Conclusiones}

V Televisión surge en un momento (2010) en el que hay noticias a diario con mucho peso informativo. Con la emisión de un programa en prime-time de análisis y tertulia Vía V se ha aprovechado de ello y ha conseguido datos de audiencia que duplican a la media del canal.

Aunque los Informativos de V Televisión nazcan con el objetivo de contar todo lo que sucede en Galicia, cerca de la mitad del programa en prime-time está dedicado a noticias nacionales e internacionales, por lo que la competencia con los espacios informativos que se emiten en su misma franja es mucho mayor. A pesar de la gran importancia que tiene la televisión como fuente informativa para la mayoría de los ciudadanos, la reducción del tiempo dedicado al telediario es acertada. Está claro que el público reclama información en este medio pero se trata de una audiencia difícil de fidelizar, ya que encuentra los mismos contenidos en otras cadenas antes que en $V$ Televisión. Por otra parte, la idea de apostar por un espacio informativo de tertulia y análisis en prime-time (Vía $\mathrm{V}$ ) resulta más atractiva para un público que ya no busca la instantaneidad informativa en televisión sino en otros medios digitales.

El estudio demuestra que existe una relación directa entre el tráfico generado en redes sociales y la audiencia televisiva que alcanza el programa en prime-time de $V$ Televisión. Cuanto mayor sea la actividad total (número de entradas, de "me gustas" y de comentarios publicados) en Facebook mayor será el número de espectadores que capte el formato. En Twitter ocurre lo mismo, cuanto mayor es la actividad y la capacidad del programa de crear conversación en la red, mayor es la cuota de pantalla que alcanza.

A través de estos nuevos medios los espectadores se convierten en protagonistas del debate al formularse en directo las preguntas que ellos publican en internet. Este método funciona especialmente en el caso de entrevistas a políticos, ya que el programa permite que el público ejerza su derecho a opinar sobre ellos y que estos le contesten.

\section{Bibliografía}

AIMC: Estudio General de los Medios para el $3^{\circ}$ Año Móvil 2012. Disponible en: http://www.aimc.es/spip.php?action=acceder_document\&arg $=2222 \& \mathrm{cle}=\mathrm{a} 2 \mathrm{f}-$ 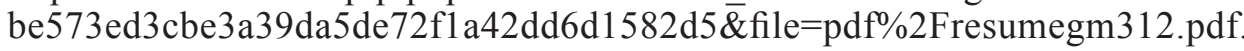 [13-10-2013]

BARLOVENTO COMUNICACIÓN: Análisis televisivo 2012. Disponible en: http:// www.barloventocomunicacion.es/images/publicaciones/ANALISIS\%20TELEVISIVO\%202012\%20ao\%20completo.pdf. [13-10-2013] 
BUSTAMANTE, Enrique (2011): "Los informativos en televisión y el espacio público democrático". En CASERO RIPOLLÉS, Andreu (2011). Periodismo en televisión. Nuevos horizontes, nuevas tendencias. Comunicación social.

CASERO RIPOLLÉS, Andreu (2011). Periodismo en televisión. Nuevos horizontes, nuevas tendencias. Comunicación social.

DOG. No 171, el 6 de septiembre de 2005. Disponible en: http://www.xunta.es/dog/ Publicados/2005/20050906/Anuncio2202E_gl.html. [13-10-2013]

GONZÁLEZ OÑATE, C. (2008): Nuevas estrategias de televisión. El desafio digital. Identidad, marca y continuidad televisiva. Madrid: Ediciones de las Ciencias Sociales.

MIGUEL DE BUSTOS, Juan Carlos y CASADO DEL RÍO, Miguel Ángel (2012): Televisiones Autonómicas. Evolución y crisis del modelo público de proximidad. Gedisa.

NOGUERA, J.M. (2010). "Redes sociales como paradigma periodístico. Medios españoles en Facebook". En: Revista Latina de Comunicación Social, n65. p. 176-186.

ORTELLS, S. (2012). "Información televisiva y redes sociales: nuevas vías para la distribución de contenidos audiovisuales". En LEÓN, B (2012). La televisión ante el desafio de internet. Comunicación Social Ediciones y Publicaciones.

ROMÁN PORTAS, Mercedes (2012). "TDT en España y el dividendo digital". En: Estudios sobre el mensaje periodístico, vol. 18, $\mathrm{n}^{\circ}$ especial noviembre, Madrid: Servicio de Publicaciones de la Universidad Complutense. p. 801-809. Disponible en: http://revistas.ucm.es/index.php/ESMP/article/view/40959. [13-10-2013]

\section{Las autoras}

Alba López Bolás: Doctoranda en Comunicación por la Universidad de Vigo. Licenciatura en Comunicación Audiovisual (2011). Máster en Edición Periodística de La Voz de Galicia (2012). Máster en Investigación en Comunicación por la Universidad de Vigo (2013).

Mercedes Román Portas: Directora de la Sección Departamental de Comunicación del Departamento Psicología Evolutiva y Comunicación de la Universidad de Vigo. Doctora en Periodismo por la Universidad de Navarra (1994). Profesora titular de periodismo en la Universidad de Vigo (1998). En la actualidad imparte docencia en el Grado en Publicidad y Relaciones Públicas y en el Grado de Comunicación Audiovisual de la Universidad de Vigo: Historia de la Comunicación y Teoría de la Comunicación. 